Rev. salud pública. 13 (6): 980-989, 2011

\title{
Hemoglobina glicosilada A1c vs. glucemia plasmática en ayunas de pacientes ambulatorios de un laboratorio médico
}

\section{Glycosylated haemoglobin A1c compared to fasting plasma glucose in outpatients referred to a medical laboratory}

María Isabel Múnera-Jaramillo ${ }^{1}$, Mary Alejandra Restrepo-Lozada ${ }^{1}$, Lina María Gómez-

Bahamón ${ }^{1}$, Doris del Rosario Mesa-Suarez ${ }^{1}$,

Blanca Susana Ramirez-Puerta²

1 Laboratorio Médico Echavarría (LME). Medellín, Colombia. mmunera@labechavarria.com, arestrepo@labechavarria.com, lgomez@labechavarria.com,dmesa@labechavarria.com

2 Universidad de Antioquia. Medellín, Colombia. zerimar761@gmail.com

Recibido 23 Febrero 2011/Enviado para Modificación 4 Septiembre 2011/Aceptado 7 Diciembre 2011

\section{RESUMEN}

Objetivo Comparar la prueba hemoglobina glicosilada (HbA1c) en diferentes puntos de corte, con la prueba de glucemia plasmática en ayunas (GPA); para medición de glucosa en sangre en pacientes ambulatorios de un laboratorio médico de la ciudad de Medellín, entre marzo y abril de 2010.

Métodos Estudio en 1016 muestras de sangre de pacientes referidos a un laboratorio médico. Se obtuvo la concordancia entre las pruebas (Índice de Kappa), se calcularon sensibilidad y especificidad, además se evaluaron diferentes puntos de corte para la prueba $\mathrm{HbA} 1 \mathrm{c}$ con la curva ROC. Se usó correlación de Spearman para establecer la asociación entre GPA y HbA1c.

Resultados El valor promedio de $\mathrm{HbA} 1 \mathrm{c}$ fue $5,7 \% \pm 0,8$ y de la GPA fue $96 \mathrm{mg} /$ $\mathrm{dL} \pm 26,1$; y esta última fue mayor en hombres $(99,04 \pm 32,7)$. El $75,4 \%$ de las muestras fueron normales para diagnóstico de diabetes con la prueba GPA, y con la HbA1c el 51,4 \% clasificaron en este rango. El punto de corte donde la combinación sensibilidad-especificidad presenta los mejores valores para $\mathrm{HbA} 1 \mathrm{c}$ fue $6,2 \%$.

Discusión La prueba $\mathrm{HbA} 1 \mathrm{c}$ presentó valores altos de sensibilidad y especificidad, por lo que su uso rutinario en el diagnóstico de diabetes mellitus podría contribuir a la búsqueda activa y la detección precoz de casos, que aseguren un mejor control de los factores de riesgo.

Palabras Clave: Hemoglobina A Glicosilada, glucemia; hiperglucemia; Diabetes mellitus, glucosa (fuente: DeCS, BIREME). 


\section{ABSTRACT}

Objective Comparing haemoglobin A glycosylate (HbA1c) at different cutoff points for blood glucose measurement to the fasting plasma glucose (FPG) test in outpatients visiting a medical laboratory in the city of Medellin between March and April 2010.

Methods 1,016 blood samples were studied from patients who had been referred to a medical laboratory. Agreement was obtained between tests (Kappa index); sensitivity and specificity were calculated. Different cutoff points for the HbA1c test were also evaluated with the ROC curve. Spearman correlation was used to establish association between FPG and $\mathrm{Hb} \mathrm{A1c}$.

Results Average $\mathrm{HbA} 1 \mathrm{c}$ was $5.7 \% \pm 0.8$ and average FPG was $96 \mathrm{mg} / \mathrm{dL} \pm 26.1$; this was higher in men $(99.04 \pm 32.7) .75 .4 \%$ of the samples came within the normal range for diagnosing diabetes with the FPG test, compared to $51.4 \%$ with the $\mathrm{HbA} 1 \mathrm{c}$ test. The cutoff point where combined sensitivity and specificity presented the best $\mathrm{HbA} 1 \mathrm{c}$ value in this study group was $6.2 \%$.

Discussion The HbA1c test had high sensitivity and specificity values, so its routine use in diagnosing diabetes mellitus could contribute towards active and early detection of cases to ensure better control of risk factors.

Key Words: Glycosylated haemoglobin A, blood glucose, hyperglycaemia, diabetes mellitus, glucose (source: MeSH, NLM).

$\mathrm{L}$ a Diabetes Mellitus (DM) constituye un grupo de enfermedades metabólicas que se caracteriza por hiperglucemia, y cuando es crónica se asocia con deterioro en el tiempo, disfunción y falla de órganos, especialmente ojos, riñones, nervios, corazón, y vasos sanguíneos (1). La DM se presenta por defectos en la secreción y/o en la acción de la insulina, que originan diferentes formas de DM, entre las cuales la tipo 2 es la de más alta prevalencia y se caracteriza por presentar resistencia a la insulina, por lo tanto el organismo es incapaz de utilizarla eficazmente.

Por su alta prevalencia, la diabetes mellitus (DM) tipo 2 se reconoce como un problema de salud pública, que está en aumento debido a factores como el envejecimiento poblacional, incremento de la prevalencia de sobrepeso y sedentarismo (2). La tendencia en el incremento del problema en la población ha sido evidenciada por la Federación Internacional de Diabetes (IFD), que en 2003 reportó 194 millones de personas diabéticas en el mundo; y se calcula que para el 2025 la cifra llegue a los 300 millones de personas afectadas (3). 
En el país las estadísticas son igualmente preocupantes, en 2007 la Encuesta Nacional de Salud encontró una prevalencia de diabetes referida por entrevista en la población general de 18 a 69 años de 3,5\%, aunque disminuyó a 2,5 \% cuando se hizo la estimación mediante la toma de una muestra de sangre capilar en una submuestra de la población de estudio; cifra superior a la reportada en 1998 que fue de 2,0\% $(4,5)$. Por otro lado la Asociación Colombiana de Diabetes (ACD) reportó que el 7,0 \% de la población colombiana mayor de 30 años y residente en área urbana tiene DM tipo 2, y anualmente se reportan de 3 a 4 casos del tipo 1 por cada 100.000 niños menores de 15 años (6), que es el tipo de diabetes más frecuente en niños y adolescentes y representa cerca del 10,0\% de todos los tipos de diabetes; mientras que el 90,0 \% de las personas con diabetes en el mundo tienen el tipo 2. Esta última se caracteriza porque los síntomas son menos severos y por lo tanto en sus primeras etapas es asintomática, en consecuencia se detecta varios años después de su inicio, cuando aparecen las complicaciones (7).

Las consecuencias en el deterioro del organismo debido a un diagnóstico tardío, soportan la necesidad de avanzar en definir un método diagnóstico oportuno, con detección de casos antes de la ocurrencia del deterioro de órganos blanco. En la actualidad los criterios diagnósticos de DM se basan en un valor mayor o igual de 6,5 \% de HbA1c, el valor de la GPA mayor o igual de $126 \mathrm{mg} / \mathrm{dl}(7,0 \mathrm{mmol} / \mathrm{l})$ o el valor a las dos horas tras la ingesta de 75 gramos de glucosa mayor o igual de $200 \mathrm{mg} / \mathrm{dl}$ (11,1 mmol/1), o también en el caso de presentar un valor superior a $200 \mathrm{mg} / \mathrm{dL}$ al azar y síntomas compatibles de DM. (1)

Recientemente un comité internacional de expertos, integrado por miembros designados de la Asociación Americana de Diabetes, la Asociación Europea para el Estudio de la Diabetes, y la Federación Internacional de Diabetes, recomendó la inclusión de la HbA1c para el diagnóstico inicial de diabetes. El Comité estableció que se hace diagnóstico de diabetes tipo 2 a cualquier persona con un valor de HbAlc confirmado $\geq 6,5 \%$ (48 $\mathrm{mmol} / \mathrm{mol})$, sin pruebas de glucosa, aunque en personas en las que la medida de HbAlc puede ser inadecuada (VIH, personas mayores de 65 años, hemoglobinopatías, gestantes), los criterios de GPA seguiran siendo utilizados (8). 
Entre las ventajas que plantea el Comité de expertos de la $\mathrm{HbA} 1 \mathrm{c}$ frente a la GPA y prueba de tolerancia oral a la glucosa postprandial, es que no requiere ayuno, ofrece un resultado estimado de la concentración de glucosa en el eritrocito de 120 días anteriores a la toma de la muestra, no requiere preparaciòn previa del paciente; razón por la cual algunos autores proponen utilizar la HbAlc como marcador de seguimiento y como método diagnostico, ya que en condiciones normales, refleja la concentración de glucosa en sangre en un periodo de 2 a 3 meses anteriores al análisis (9).

El presente estudio se realizó con el objetivo de identificar en pacientes ambulatorios el comportamiento de la HbAlc con la GPA, en las condiciones de uso de rutina en un laboratorio médico y evaluar diferentes puntos de corte de la prueba.

\section{MÉTODOS}

Se incluyeron 1016 pacientes ambulatorios, quienes fueron remitidos a un laboratorio médico para realizarse por solicitud médica examen de GPA, en el periodo comprendido entre marzo y abril de 2010, se excluyeron del estudio mujeres embarazadas. Las muestras de sangre fueron tomadas en los puntos de servicio del laboratorio, siguiendo normas estrictas de bioseguridad y se analizaron en la Sección de Química de la Central de Procesamiento. Siguiendo las directrices de la resolución 8430 de 1993 del Ministerio de salud (10), el proyecto fue aprobado por el Comité de ética del laboratorio médico donde se realizó el estudio y se garantizó la confidencialidad de la información.

A todas las muestras se les hizo cuantificación de HbA1c y GPA. Para la medición de glucosa en sangre, se utilizó el método glucosa oxidasa y las muestras se procesaron en el Analizador Advia 1200 (Siemens Medical Solutions Diagnostics). La cuantificación de la hemoglobina glicosilada, se realizó con el método de inmunoensayo, para lo cual se utilizó el equipo DCA 2000 + (Siemens Healthcare Diagnostics), método que ha sido certificado por National Glycohemoglobin Standarization Program (NGSP) y estandarizado por Diabetes Complication and Control Trial (DCCT). (11)

Para el análisis de los datos, se hizo una categorización del grupo de estudio según el resultado de ambas pruebas de la siguiente manera: normoglucémicos (glucemia basal $<100 \mathrm{mg} / \mathrm{dl}$ y $\mathrm{HbAlc}<5,7 \%$ ), pre- 
diabéticos (glucemia basal de 100 - $125 \mathrm{mg} / \mathrm{dl}$ y HbA1c de 5,7 - 6,4 \%) y diabéticos (glucemia basal $\geq 126 \mathrm{mg} / \mathrm{dl}$ y HbAlc $\geq 6,5 \%$ ); de acuerdo con la clasificación de la Asociación Americana de Diabetes (ADA). (1)

Los datos se analizaron en SPSS ${ }^{\circ}$, versión 18.0 y Epidat 3.1. Para las variables demográficas y los resultados globales de las pruebas se obtuvieron distribuciones de frecuencia, promedios y desviaciones estándar. En la comparación de las dos pruebas se calcularon sensibilidad, especificidad, valores predictivos positivos y negativos y se estableció la concordancia entre las dos pruebas (Índice de Kappa); además se evaluaron diferentes puntos de corte para la HbAlc con la curva ROC. Se usó correlación de Spearman para establecer la asociación entre GPA y HbA1c y se realizó un gráfico de dispersión entre los valores de las dos pruebas.

\section{RESULTADOS}

Se estudiaron en total 1016 pacientes, de los cuales el 66,6 \% eran del género femenino, y la edad media del grupo de estudio fue 52,3 años \pm 18,7 . El valor promedio de $\mathrm{HbA} 1 \mathrm{c}$ fue $5,7 \% \pm 0,8$. Las medianas fueron 5,6 $\%$ en hombres y $5,7 \%$ en mujeres.

El valor promedio de la GPA fue $96 \mathrm{mg} / \mathrm{dL} \pm 26,1$, y fue mayor en hombres $(99,04 \mathrm{mg} / \mathrm{dL} \pm 32,7)$, que en mujeres $(94,47 \mathrm{mg} / \mathrm{dL} \pm 22)$. Las medianas fueron $94 \mathrm{mg} / \mathrm{dL}$ y $90 \mathrm{mg} / \mathrm{dL}$ respectivamente, encontrándose diferencias estadísticamente significativas (prueba U de Mann-Whitney, valor $\mathrm{p}<0,001)$.

La distribución del grupo de estudio de acuerdo con del estado glucémico por tipo de examen, muestra que $75,4 \%$ (766) estaban en el rango normal para GPA (Tabla 1); en tanto que 51,4\% (522) clasificaron en este rango con la prueba $\mathrm{HbAlc}$.

El índice de Kappa cuando se incluyeron las tres categorías fue moderado, 0.44 (IC $95 \% \quad 0,38-0,50$ ) y cuando el grupo de estudio fue clasificado solo en dos categorías, diabéticos y no diabéticos el valor de Kappa fue 0,51 (IC $95 \% 0,40-0,62$ ). 
En la Tabla 2 se presentan los hallazgos de sensibilidad, especificidad y valores predictivos, con sus respectivos intervalos de confianza, para la evaluación de la $\mathrm{HbA1c}$ frente a la GPA en diferentes puntos de corte a partir de $6,0 \%$ hasta $6,7 \%$.

Tabla 1. Clasificación del estado glucémico del grupo de estudio con las pruebas GPA y $\mathrm{HbA} 1 \mathrm{c}$

\begin{tabular}{|c|c|c|c|c|}
\hline \multirow[b]{3}{*}{ Estado Glicémico } & \multicolumn{4}{|c|}{ Prueba Diagnóstica } \\
\hline & \multicolumn{2}{|c|}{$\mathrm{GPA}^{*}$} & \multicolumn{2}{|c|}{$\mathrm{HbA} 1 \mathrm{c}^{* \star}$} \\
\hline & $n$ & $\%$ & $\mathrm{~N}$ & $\%$ \\
\hline $\begin{array}{l}\text { Normal } \\
{ }^{*}<100 \mathrm{mg} / \mathrm{dl} \\
*<5,7 \%\end{array}$ & 766 & 75,4 & 522 & 51,4 \\
\hline $\begin{array}{l}\text { Prediabético } \\
{ }^{*} 100-125 \mathrm{mg} / \mathrm{dl} \\
{ }^{* *} 5,7-6,4 \%\end{array}$ & 203 & 20,0 & 415 & 40,8 \\
\hline $\begin{array}{l}\text { Diabético } \\
{ }^{*}>=126 \mathrm{mg} / \mathrm{dl} \\
{ }^{* *}>=6,5 \%\end{array}$ & 47 & 4,6 & 79 & 7,8 \\
\hline Total & 1016 & 100 & 1016 & 100 \\
\hline
\end{tabular}

Tabla 2. Sensibilidad, especificidad y valores predictivos (IC $95 \%$ ) para la detección de diabetes en diferentes puntos de corte de HbA1c, en comparación con la prueba de GPA

\begin{tabular}{ccccc}
\hline & Sensibilidad\% & Especificidad\% & VPP\% & VPN\% \\
\hline $\mathrm{HbA}_{1 \mathrm{c}} \%$ & & & & \\
6,0 & $85,1(73,9-96,4)$ & $80,1(77,5-82,7)$ & $17,2(12,1-22,2)$ & $99,1(98,4-99,8)$ \\
6,1 & $83,0(71,2-94,8)$ & $85,8(83,5-88,0)$ & $22,0(15,7-28,4)$ & $99,1(98,3-99,8)$ \\
6,2 & $83,0(71,2-94,8)$ & $89,6(87,6-91,6)$ & $27,9(20,1-35,6)$ & $99,1(98,4-99,8)$ \\
6,3 & $80,9(68,5-93,2)$ & $91,9(90,1-93,6)$ & $32,5(23,6-41,4)$ & $99,0(98,3-99,7)$ \\
6,4 & $78,7(66,0-91,5)$ & $93,8(92,2-95,4)$ & $38,1(28,0-48,3)$ & $98,9(98,2-99,6)$ \\
6,5 & $72,3(58,5-86,2)$ & $95,4(94,0-96,7)$ & $43,0(31,5-54,6)$ & $98,6(97,8-99,4)$ \\
6,7 & $66,0(51,4-80,6)$ & $97,0(95,9-98,1)$ & $51,7(38,2-65,1)$ & $98,3(97,5-99,2)$ \\
\hline
\end{tabular}

Según el análisis de curvas de características operativas para el receptor (ROC), el punto de corte donde la combinación sensibilidad-especificidad presenta los mejores valores para HbAlc, en el grupo de estudio fue 6,2 \%; con un área por debajo de la curva de 0,914 (IC $95 \%$ 0,86 - 0,96) (Figura 1).

Tanto la prueba de glucemia basal como la de hemoglobina glicosilada, no presentaron distribución normal según prueba de normalidad de Kolmogorov-Smirnov. El análisis de correlación entre las mediciones de GPA y HbA1c mostró un coeficiente correlación de Spearman de 0,48. (Figura 2). 
Figura 1. Curva ROC de HbA1c para detectar diabetes en diferentes puntos de corte

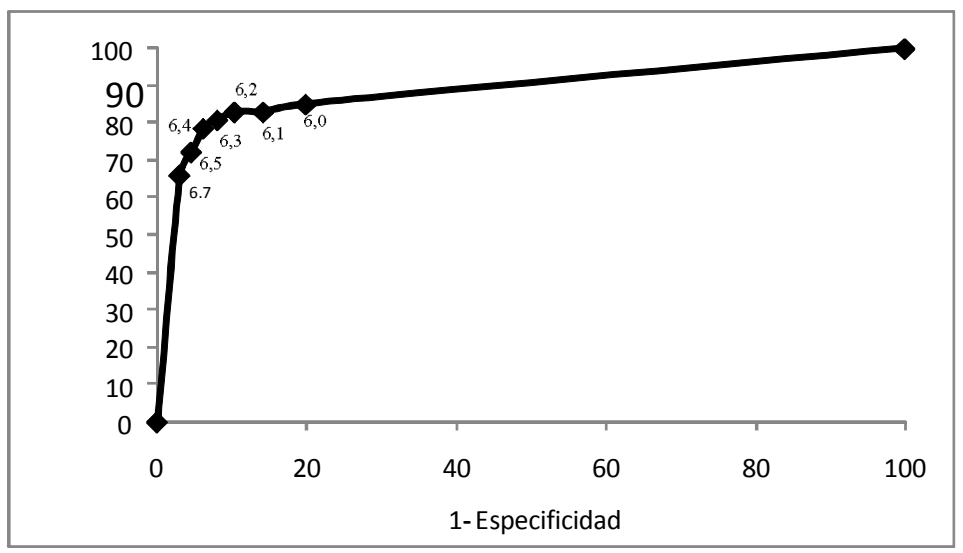

Figura 2. Correlación entre las pruebas diagnósticas GB y HbA1c

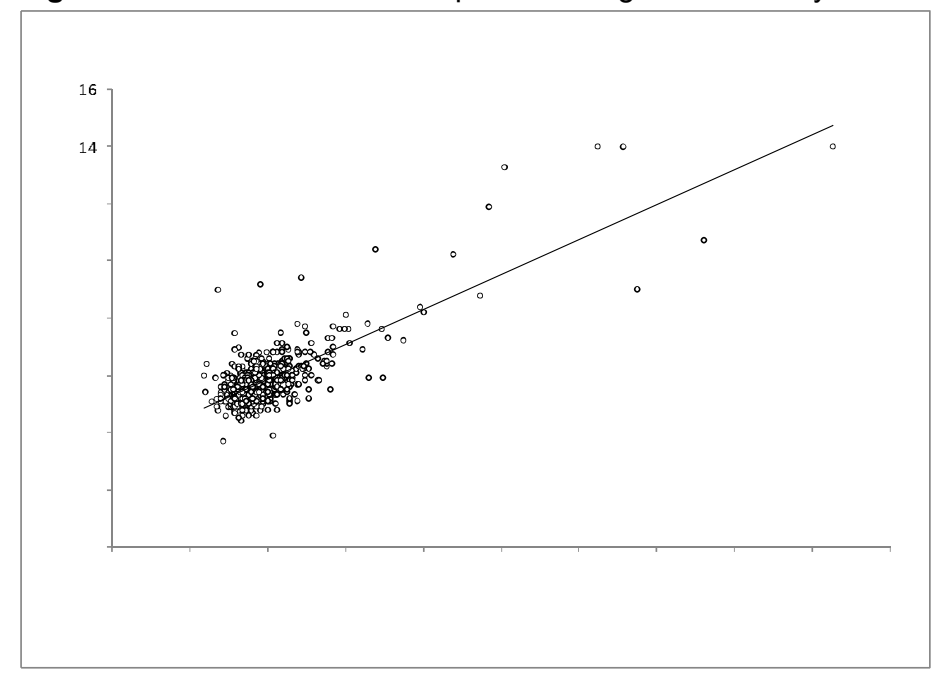

\section{DISCUSIÓN}

En Colombia en 1999, el estudio nacional de factores de riesgo de enfermedades crónicas (ENFREC II), reportó una prevalencia de diabetes mellitus en población general del 2,0 \% (5). Diez años después, en el 2007, la prevalencia se incrementó al 2,6 \% (4), esta situación refleja la importancia de la búsqueda activa y la detección precoz de casos. (5) 
Teniendo en cuenta que al clasificar los pacientes por estado glicémico con la prueba $\mathrm{HbAlc}$, con base en el punto de corte recomendado por la OMS (6,5\%), se encontró un 7,8\% de pacientes en el rango de diabetes; mientras que con la GPA se detectaron solo 4,6 \%; lo que indica que la prueba $\mathrm{HbA} 1$ contribuiría a identificar más casos de pacientes diabéticos o en riesgo de diabetes.

Los hallazgos del presente estudio en la evaluación de la HbAlc en comparación con la GPA, aportan evidencia acerca de la utilidad de la prueba para el tamizaje y diagnóstico de diabetes mellitus en población de riesgo, dado que la evaluación de la prueba se hizo en pacientes a quienes por orden médica se les había hecho GPA. En el presente estudio no se encontraron diferencias estadìsticamente significativas en los valores promedio de la concentración de HbAlc según género; este hallazgo fue similar al reportado por Selvin y colaboradores del departamento de epidemiología de Johns Hopkins (12).

En cuanto al valor de referencia para la concentración de glucosa en sangre con base en la prueba HbAlc, la Organización Mundial de la Salud recomienda como punto de corte para diagnóstico de diabetes $\geq 6,5 \%$, pero advierte que un valor inferior no excluye diabetes (13). En un estudio publicado por Bao Yuquian y colaboradores, en población general china, el punto de corte con mejor desempeño para la prueba de HbAlc fue 6,3 \% (14); este hallazgo fué similar al encontrado en pacientes ambulatorios y en el presente estudio $6,2 \%$.

Adicionalmente, la concordancia obtenida entre las dos pruebas diagnósticas fue moderada, situación que podría ser explicada porque las dos pruebas miden condiciones diferentes en el tiempo y en la molécula que es objeto de análisis. Por un lado la prueba de GPA mide solo la concentración de glucosa en plasma en un momento dado y la HbAlc mide el valor promedio de los últimos dos a tres meses de esta molécula; así mismo, mientras la HbAlc mide la cantidad de glucosa unida a la hemoglobina, la prueba de GPA mide la concentración de este carbohidrato en plasma mediante una reacción enzimática (15). Este hallazgo es similar al de un estudio realizado en población general en China, publicado por Woo-Jun Yun y otros, quienes tambien reportaron una concordancia moderada, con un Kappa de 0,50 (16). 
En Colombia la medición de HbA1c hace parte de las pruebas de rutina de laboratorio clínico y está incluida en el plan obligatorio de salud según el acuerdo 008 de la Comisión de Regulación en Salud-CRES (17), pero solo se usa para el control de pacientes a quienes ya se les ha diagnósticado diabetes. Adicionalmente, varios de los métodos utilizados en los laboratorios del país para la cuantificación de la HbA1c están certificados bajo los estándares de la National Glycohemoglobin Standardization Program -NGSP- (11). Por lo tanto en el país en general los laboratorios clínicos tienen las condiciones que les permiten cumplir con la mayoria de las recomendaciones de la OMS para implementar la HbA1c en el diagnóstico inicial de diabetes (15).

Los resultados de este estudio permitieron identificar el desempeño de la prueba de HbA1c frente a la GPA en pacientes remitidos para examen de GPA, que pudieran ser considerados como población en riesgo, así mismo se exploró un posible punto de corte en el grupo de estudio. La realización de estudios similares comparando HbA1c con GPA en el rango de 5,7-6,4 $\%$ y 100-125 mg/dl respectivamente, aportarían información adicional en el riesgo de padecer diabetes en pacientes que presenten intolerancia a la glucosa.

Debe tenerse en cuenta que las muestras analizadas en este estudio, eran de pacientes remitidos por el médico tratante para hacerse exámenes de GPA; no se disponía de información acerca de la condición de salud de los pacientes, lo que limita el análisis de las interferencias que pudieran presentarse en la prueba $\mathrm{HbA1c}$; por lo tanto los hallazgos de este estudio no son concluyentes respecto al comportamiento de la $\mathrm{HbAlc}$, aunque si constituyen un acercamiento en este aspecto.

La prueba HbA1c presentó valores altos de sensibilidad y especificidad, por lo que su uso rutinario en el diagnóstico de diabetes mellitus podría contribuir a la búsqueda activa y la detección precoz de casos, que aseguren un mejor control de los factores de riesgo; se requiere por lo tanto avanzar en la realización de estudios controlados que permitan definir de manera más precisa el comportamiento de la prueba en la población colombiana

Agradecimientos: Al Laboratorio Médico Echavarría (LME), por su aporte en la financiación del estudio y a la empresa Siemens Health Care quien donó los reactivos para realizar las pruebas de $\mathrm{HbAlc}$. Igualmente los autores agradecen a la Dra. Catalina Pérez Colle por su acompañamiento en la planeación del presente estudio. 


\section{REFERENCIAS}

1. American Diabetes Association. Diagnosis and classification of diabetes mellitus. Diabetes Care 2010; 33:S62-S68.

2. Organización Panamericana de la Salud. Iniciativa de la diabetes para las américas (DIA): Plan de acción para América Latina y el Caribe 2001-2006; 2001 Jul. Reporte No : OPS/HCP/HCN/01.05.

3. International Diabetes Federation. A world-wide call to action. The DAWN International Expert Advisory Board. Diabetes Voice 2004; 49: 12-13.

4. República de Colombia. Ministerio de la Protección Social. Instituto Colombiano para el Desarrollo de la Ciencia y la Tecnología. Sistemas Especializados de Información. Encuesta Nacional de Salud 2007.

5. Colombia. Ministerio de Salud. Centro Nacional de Consultoría. Estudio Nacional de Salud Bucal 3, 1999, Colombia. Estudio Nacional de Factores de Riesgo de Enfermedades Crónicas 2. Prevalencia de diabetes mellitus y de glucosa alterada en ayunas, Tomo V. Serie Informes Técnicos. Bogotá, 1999.

6. Federación Diabetológica Colombiana [Internet]. Alerta sobre el incremento de la diabetes hace la Federación Diabetológica Colombiana. Diabetes, Control y Prevención 2007; 1:1. Disponible en: URL: http://www.fdc.org.co/Periodico/vol1pag1.html. Consultado 12 Noviembre 2010.

7. Bennett CM, Guo M, Dharmage SC. HbA1c as a screening tool for detection of type 2 diabetes: a systematic review. Diabet Med 2007; 24: 333-343.

8. American Diabetes Asociation. International Expert Committee. Report on the Role of the A1C Assay in the Diagnosis of Diabetes. Diabetes Care 2009; 32(7):1327-1334.

9. García FJ. Utilización de la hemoglobina glucosilada para el diagnóstico de diabetes. Cad Aten Primaria Año 2009 (16): 228-229.

10. República de Colombia. Ministerio de Salud. Resolución Nº 008430 del 4 de octubre de 1993, por la cual se establecen las normas científicas, técnicas y administrativas para la investigación en salud.

11. National Glycohemoglobin Standardization Program (NGSP) [Internet]. List of NGSP certified methods. 2010. Disponible en: URL: http://www.ngsp.org/ Consultado 7 Septiembre 2010.

12. Selvin E, Zhu H, Brancati FL. Elevated A1c in adults without a history of diabetes in the U.S. Diabetes Care 2009; 32(5): 828-833.

13. World Health Organization. Use of glycated haemoglobin $(\mathrm{HbA} 1 \mathrm{c})$ in the diagnosis of diabetes mellitus. Abbreviated report of a WHO consultation. Geneva; 2011. Report No. :WHO/NMH/CHP/CPM/11.1

14. Yuqian B, Xiaojing M, Huating L, Mi Z, Cheng $H$, Haiya Wu, et.al. Glycated haemoglobin $\mathrm{A} 1 \mathrm{c}$ for diagnosing diabetes in Chinese population: cross sectional epidemiological survey. BMJ 2010; 340:c2249.

15. World Health Organization and International Diabetes Federation. Screening for type 2 diabetes. Geneva; 2003.

16. Woo JY, Min HS, Sun SK, Kyeong SP, Young HL, Hae SN, et al. A comparison of fasting glucose and $\mathrm{HbA} 1 \mathrm{c}$ for the diagnosis of diabetes mellitus among Korean adults. $\mathrm{J}$ Prev Med Public Health 2010; 43(5): 451-454.

17. República de Colombia. Comisión de regulación en salud-CRES- de 2009. Acuerdo No 008 , mediante el cual se aclara y actualiza el plan obligatorio en salud-POS-, para los regímenes contributivo y subsidiado. Diciembre 29 de 2009. 